\title{
APPROXIMATION OF *-NONEXPANSIVE RANDOM MULTIVALUED OPERATORS ON BANACH SPACES
}

\author{
ISMAT BEG, A. R. KHAN and N. HUSSAIN
}

(Received 14 February 2001; revised 8 January 2003)

Communicated by A. Pryde

\begin{abstract}
We establish the existence and approximation of solutions to the operator inclusion $y \in T y$ for deterministic and random cases for a nonexpansive and *-nonexpansive multivalued mapping $T$ defined on a closed bounded (not necessarily convex) subset $C$ of a Banach space. We also prove random fixed points and approximation results for ${ }^{*}$-nonexpansive random operators defined on an unbounded subset $C$ of a uniformly convex Banach space.
\end{abstract}

2000 Mathematics subject classification: primary 47H40, 47H10, 41 A65, 47H09, 60H25.

Keywords and phrases: random fixed point, *-nonexpansive random multivalued map, weakly inward operator, Leray-Schauder condition, Opial condition, random approximation, Banach space.

\section{Introduction and preliminaries}

Probabilistic functional analysis is an important mathematical discipline because of its applications to probabilistic models in applied problems. Random operators lie at the heart of this discipline and their theory is needed for the study of various classes of random equations. The study of random fixed points and random approximations have gained tremendous importance after the publication of papers by Beg [2], Beg and Shahzad [4-6], Lin [14, 15], Papageorgiou [17], Sehgal and Singh [19, 20], Tan and Yuan [23-25] and Xu [26].

In this paper the notions of *-nonexpansive and weakly nonexpansive multivalued maps with values which are not subsets of the domain are introduced. In Section 2, the existence and approximation of solutions to the nonlinear operator inclusion $y \in T y$ is discussed. Section 3 deals with the approximation of solutions to the nonlinear random operator inclusion $y(\omega) \in T(\omega, y(\omega))$ for each $\omega \in \Omega$, where $(\Omega, A)$ is a measurable 
space. In Section 4, we establish a random fixed point theorem for *-nonexpansive multivalued operators (which are not continuous) defined on closed convex subsets of a Banach space. A random approximation theorem for ${ }^{*}$-nonexpansive multivalued random operator defined on unbounded subsets of a Hilbert space is also proved.

Throughout this paper $(\Omega, A)$ denotes a measurable space with $\boldsymbol{A}$ a sigma algebra of subsets of $\Omega$. Let $C$ be a subset of a normed space $X$. We denote by $2^{X}, C(X)$, $C K(X), K(X)$ and $C B(X)$ the families of all nonempty, nonempty closed, nonempty convex compact, nonempty compact and nonempty closed bounded subsets of $X$ respectively. A mapping $T: \Omega \rightarrow 2^{X}$ is called measurable if for any open subset $B$ of $X, T^{-1}(B)=\{\omega \in \Omega: T(\omega) \cap B \neq \phi\} \in A$. A mapping $T: C \rightarrow 2^{X}$ is upper (lower) semicontinuous if for any closed (open) subset $B$ of $X, T^{-1}(B)$ is closed (open); if $T$ is both upper and lower semicontinuous, then $T$ is called a continuous map. A mapping $\xi: \Omega \rightarrow X$ is said to be a measurable selector (see, for example, [8]) of a measurable mapping $T: \Omega \rightarrow 2^{X}$ if $\xi$ is measurable and for any $\omega \in \Omega, \xi(\omega) \in T(\omega)$. A mapping $T: \Omega \times C \rightarrow 2^{X}$ is a random operator if for any $x \in C, T(\cdot, x)$ is measurable. A mapping $\xi: \Omega \rightarrow X$ is said to be a generalized fixed point of a random operator $T$ (generalized solution to $y(\omega) \in T(\omega, y(\omega))$ if for each $\omega \in \Omega, \xi(\omega) \in T(\omega, \xi(\omega))$; in case $\xi$ is a measurable function, it is called a random fixed point of $T$ (random solution to $y(\omega) \in T(\omega, y(\omega)$ ). For $x \in X$, let

$$
P_{C}(x)=\{y \in C:\|x-y\|=d(x, C)\}, \quad \text { where } d(x, C)=\inf _{y \in C}\|x-y\| .
$$

If $P_{C}$ is a single-valued map, it is called a proximity map. Later, we will use $P$ instead of $P_{C}$.

A multivalued map $T: C \rightarrow 2^{X}$ is said to be

(i) weakly nonexpansive if given $x \in C$ and $u_{x} \in T x$ there is a $u_{y} \in T y$ for each $y \in C$ such that $d\left(u_{x}, u_{y}\right) \leq d(x, y)$;

(ii) *-nonexpansive if for all $x, y \in C$ and $u_{x} \in T x$ with $d\left(x, u_{x}\right)=d(x, T x)$, there exists $u_{y} \in T y$ with $d\left(y, u_{y}\right)=d(y, T y)$ such that $d\left(u_{x}, u_{y}\right) \leq d(x, y)$ (see $[9,27])$;

(iii) weakly inward if $T x \subset \operatorname{cl}\left(I_{C}(x)\right)$ for all $x \in C$, where $I_{C}(x)=\{z \in X: z=$ $x+\lambda(y-x)$ for some $y \in C$ and $\lambda \geq 0$ ), is inward set of $x$ relative to $C$ and cl stands for the closure (see, for example, [28]);

(iv) hemicompact if each sequence $\left\{x_{n}\right\}$ in $C$ has a convergent subsequence whenever $d\left(x_{n}, T x_{n}\right) \rightarrow 0$ as $n \rightarrow \infty$;

(v) demicompact if each sequence $\left\{x_{n}\right\}$ in $C$ has a convergent subsequence whenever $\left\{x_{n}-T x_{n}\right\}$ is a convergent sequence in $X$;

(vi) condensing (or densifying) if for each nonempty bounded subset $B$ of $C$ with $\alpha(B)>0, T(B)$ is bounded and $\alpha(T(B))<\alpha(B)$, where

$\alpha(B)=\inf \{r>0: B$ can be covered by a finite number of sets of diameter $\leq r\}$. 
If there exists $k, 0<k \leq 1$, such that for each nonempty bounded subset $B$ of $C$ we have $\alpha(T(B)) \leq k T(B)$, then $T$ is called $k$-set-contractive map. Let $T_{\beta}: C \rightarrow 2^{X}$ be a map for each $\beta \in I$ (some indexing set). The collection $\left\{T_{\beta}: \beta \in I\right\}$ is called collectively condensing (collectively $k$-contractive) if $\alpha\left(\bigcup_{\beta} T_{\beta} B\right)<\alpha(B)$ $\left(\alpha\left(\bigcup_{\beta} T_{\beta} B\right) \leq k \alpha(B)\right)$ for all bounded subsets $B$ of $C$ (see, for example, [16]).

As in Xu [27], by $P_{T}(x)$ we mean the set $\left\{u_{x} \in T x: d\left(x, u_{x}\right)=d(x, T x)\right\}$ (possibly empty) for each $x$ in $C$.

A mapping $T: C \rightarrow C B(X)$ is a contraction if for any $x, y \in C, H(T x, T y) \leq$ $k d(x, y)$ where $H$ is the Hausdorff metric on $C B(X)$ and $0 \leq k<1$. If $k=1$, then $T$ is called a nonexpansive map.

Following Canetti, Marino and Pietramala [7], we define, for any $x, y \in X, \epsilon>0$ and $A \subseteq X$,

$$
\begin{aligned}
G(x, y ; C) & =\{z \in C:\|z-y\| \leq\|z-x\|\} \\
G(x, y ; \epsilon ; C) & =\{z \in C:\|z-y\|<\|z-x\|+\epsilon\} \\
G(x, A ; C) & =\{z \in C: \exists a \in A:\|z-a\| \leq\|z-x\|\}=\bigcup_{a \in A} G(x, a ; C) \\
G(x, A ; \epsilon ; C) & =\{z \in C: \ni a \in A:\|z-a\|<\|z-x\|+\epsilon\}=\bigcup_{a \in A} G(x, a ; \epsilon ; C) .
\end{aligned}
$$

For given $T: C \rightarrow 2^{X}$, we say that

(i) $C$ is $(K R)$-bounded with respect to $T$ (see, for example, $[13,18]$ ) if for some bounded set $A \subset C$, the set $G(A, T A ; C)=\bigcap_{\alpha \in A} G(a, T a ; C)$ is either empty or bounded;

(ii) a sequence $\left\{x_{n}\right\} \in C$ is asymptotically $T$-regular if $\lim _{n} d\left(x_{n}, T x_{n}\right)=0$;

(iii) $T$ satisfies condition (A) (see, for example, [22]) if for any sequence $\left\{x_{n}\right\}$ in $C, D \in C(C)$ such that $d\left(x_{n}, D\right) \rightarrow 0$ and $d\left(x_{n}, T x_{n}\right) \rightarrow 0$ as $n \rightarrow \infty$, then there exists $y \in D$ with $y \in T y$. (Note that every continuous hemicompact map satisfies condition (A).);

(iv) $T$ is $d$-continuous if, for any $y \in X$, the function $x \rightarrow d(y, T x)$ is continuous.

A random operator $T: \Omega \times C \rightarrow 2^{X}$ is said

(i) to be continuous (nonexpansive, hemicompact, ${ }^{*}$-nonexpansive etc.) if for each $\omega \in \Omega, T(\omega, \cdot)$ is continuous (nonexpansive, hemicompact, *-nonexpansive etc.);

(ii) to be weakly inward if for each $\omega \in \Omega, T(\omega, x) \subset \operatorname{cl}\left(I_{C}(x)\right)$ for each $x \in C$;

(iii) to satisfy the Leray-Schauder condition (in case $C$ has nonempty interior and it contains 0 ) if there is a point $z$ in the interior of $C$ (depending on $\omega$ ) such that for each $u_{y} \in T(\omega, y), u_{y}-z \neq m(y-z)$ for all $y$ in $\partial C$ (the boundary of $C$ ) and $m>1$;

(iv) to satisfy condition (A) if for any $\omega \in \Omega, T(\omega, \cdot)$ satisfies condition (A). 
THEOREM A ([28, Theorem 2.1]). Let $D$ be a closed subset of a Banach space $X$. Suppose that $T: D \rightarrow K(X)$ is a weakly inward contraction mapping with Lipschitz constant $L<1$. Then $T$ has a fixed point $x_{0}$ (that is, $x_{0} \in T x_{0}$ ).

A *-nonexpansive multivalued mapping is different from a continuous map as is clear from the following example.

EXAMPLE 1.1. Let $X=\mathbb{R}^{2}$ be equipped with Euclidean norm and

$$
C=\left\{(a, 0): \frac{1}{\sqrt{2}} \leq a \leq 1\right\} \cup\{(0,0)\} .
$$

Define $T: C \rightarrow 2^{X}$ by

$$
T(a, 0)= \begin{cases}(0,1) & \text { if } a \neq 0 \\ L=\text { the line segment }[(0,1),(1,0)] & \text { if } a=0 .\end{cases}
$$

Then $P_{T}(a, 0)=\{(0,1)\}$ for all $(a, 0) \in C$ with $a \neq 0$ and $P_{T}(0,0)=\{(1 / 2,1 / 2)\}$.

Clearly $T$ is *-nonexpansive but not a continuous multifunction [20, page 537]. Moreover, for given $x=(0,0)$ and $u_{x}=(1,0) \in T x$, there does not exist $y \neq x$ in $C$ and $u_{y} \in T y$ such that $\left|u_{x}-u_{y}\right| \leq|x-y|$. Recall that for $y \neq x$ in $C, u_{y}=(0,1)$ and $\left|u_{x}-u_{y}\right|=|(1,0)-(0,1)|=\sqrt{2}>d(x, y)$. So $T$ is not weakly nonexpansive.

REMARKS 1.2. (i) A *-nonexpansive map may not be weakly nonexpansive [9, page 389].

(ii) It follows from the definition of Hausdorff metric that a weakly nonexpansive map is nonexpansive. The converse holds for compact valued maps. For if $T: C \rightarrow$ $2^{X}$ is a compact valued nonexpansive map, then for any $x \in C$ and $u_{x} \in T x$, we can find some $u_{y} \in T y$ for all $y$ in $C$ by compactness of $T y$ such that $d\left(u_{x}, u_{y}\right) \leq$ $\sup \{d(u, T y): u \in T x\} \leq H(T x, T y) \leq d(x, y)$. It implies that $T$ is weakly nonexpansive (also see [27, Proposition 1]).

(iii) *-nonexpansiveness and nonexpansiveness are two different concepts for multivalued mappings.

Let $F=\left\{f_{A}\right\}_{A \in K(C)}$ be a family of functions form $[0,1]$ into $K(C)$ with the property that for each $A \in K(C), f_{A}(1)=A$. Such a family is said to be contractive if there exists a function $\varphi:(0,1) \rightarrow(0,1)$ such that for all $A, B \in K(C)$ and for all $t \in(0,1)$ we have $H\left(f_{A}(t), f_{B}(t)\right) \leq \varphi(t) H(A, B)$. Such a family is said to be jointly continuous if $f_{A}(t) \rightarrow f_{A 0}\left(t_{0}\right)$ in $K(C)$ whenever $t \rightarrow t_{0}$ in $[0,1]$ and $A \rightarrow A_{0}$ in $K(C)$.

Suppose that $G=\left\{f_{A}\right\}_{A \in K(C)}$ is a family of functions from $[0,1]$ into $K(C)$ with the property that for each sequence $\left\{\lambda_{n}\right\}$ in $(0,1]$, with $\lambda_{n} \rightarrow 1$ as $n \rightarrow \infty$ we have

$$
f_{A}\left(\lambda_{n}\right)=\lambda_{n} A \text {. }
$$


We observe that $G \subseteq F$ and it has the property of contractiveness and joint continuity. Notice that the condition (*) is satisfied by a starshaped set with 0 star centre (for more details see $[1,12])$.

\section{Approximation of multivalued operators}

THEOREM 2.1. Let $C$ be a nonempty closed and bounded subset of a Banach space $X$ and $T: C \rightarrow K(X)$ weakly inward nonexpansive mapping. Suppose that $C$ has a family $G$ satisfying condition (*). Then there exists a sequence $\left\{x_{n}\right\}$ in $C$ such that $x_{n} \rightarrow y \in C$ and $y \in T y$, provided one of the following conditions holds:

(a) Each asymptotically $T$-regular sequence in $C$ is Cauchy.

(b) $T$ is hemicompact.

(c) The collection $\left\{F_{n}: F_{n}=\lambda_{n} T, \lambda_{n} \in(0,1)\right.$ and $\left.\lambda_{n} \rightarrow 1\right\}$ is collectively condensing.

(d) The collection $\left\{F_{n}: F_{n}=\lambda_{n} T, \lambda_{n} \in(0,1)\right.$ and $\left.\lambda_{n} \rightarrow 1\right\}$ is collectively $k$ contractive where $0 \leq k<1$.

PROOF. Let $r_{n}=1-1 /(n+1)$ for each $n \geq 1$. Define $T_{n}: C \rightarrow K(X)$ by $T_{n}(x)=f_{T_{x}}\left(r_{n}\right)=r_{n} T(x)$ for all $x \in C$. Each $T_{n}$ is a weakly inward contraction map. So by Theorem $A$, there is a sequence $\left\{x_{n}\right\}$ in $C$ such that $x_{n} \in T_{n}\left(x_{n}\right)$ for $n \geq 1$. Therefore, there is $y_{n} \in T\left(x_{n}\right)$ such that $x_{n}=r_{n} y_{n}$. It now follows by the boundedness of $C$ that

$$
x_{n}-y_{n}=\left(\frac{1}{r_{n}}-1\right) x_{n} \rightarrow 0 \quad \text { as } n \rightarrow \infty .
$$

By (1), $\left\|x_{n}-y_{n}\right\|=\left(1 / r_{n}-1\right)\left\|x_{n}\right\| \rightarrow 0$ as $n \rightarrow \infty$. The set $\left\{\left\|x_{n}\right\|: n \geq 1\right\}$ is bounded so

$$
\begin{aligned}
0 & \leq d\left(x_{n}, T x_{n}\right)=\inf \left\{\left\|x_{n}-y_{n}\right\|: y \in T x_{n}\right\} \\
& \leq\left\|x_{n}-y_{n}\right\| \leq\left(\frac{1}{r_{n}}-1\right)\left\|x_{n}\right\| \rightarrow 0 \text { as } n \rightarrow \infty .
\end{aligned}
$$

Thus $\left\{x_{n}\right\}$ is an asymptotically $T$-regular sequence in $C$.

(a) Since $\left\{x_{n}\right\}$ is a Cauchy sequence in $C$ so it should converge to $y \in C$. As $T: C \rightarrow K(X)$ is continuous so for all $x, y \in C,|d(x, T x)-d(y, T y)| \leq d(x, y)+$ $H(T x, T y)$, which implies that the function $x \rightarrow d(x, T x)$ is continuous. Hence $0=\lim _{n} d\left(x_{n}, T x_{n}\right)=d(y, T y)$. It further gives that $y \in T y$.

(b) Since $\left(x_{n}, T x_{n}\right) \rightarrow 0$ as $n \rightarrow \infty$, by the definition of hemicompactness of $T$, $\left\{x_{n}\right\}$ has a convergent subsequence. Without loss of generality assume its the whole sequence and $x_{n} \rightarrow y$ in $C$. This together with (a) implies that $y \in T y$. 
(c) Define the map $F: C \rightarrow 2^{x}$ by $F x=\bigcup_{n} T_{n} x, x \in C$. For any subset $B$ of $C$,

$$
\alpha(F B)=\alpha\left(\bigcup_{x \in B} F x\right)=\alpha\left(\bigcup_{x \in B}\left(\bigcup_{n} T_{n} x\right)\right)=\alpha\left(\bigcup_{n} T_{n} B\right)<\alpha(B) .
$$

It follows by [23, Lemma 2.1] that $F$ is hemicompact. Further, $d\left(x_{n}, F x_{n}\right)=0$ for each $n=1,2, \ldots$, and hence $\left\{x_{n}\right\}$ has a convergent subsequence. Without loss of generality assume its the whole sequence and $x_{n} \rightarrow y \in C$. Thus by (a), $y \in T y$.

(d) The mapping $F: C \rightarrow 2^{X}$ defined by $F x=\bigcup_{n} T_{n} x$ is $k$-contractive and hence condensing. The result follows from (c).

THEOREM 2.2. Let $C, X, G$ and $T$ be as in Theorem 2.1. Suppose the following condition holds: $C \cap D \neq \phi$ for all $D \in K(X)$ with $D=\lim T x_{n}$ for some asymptotically $T$-regular sequence $\left\{x_{n}\right\}$ in $C$ and $T$ satisfies for all $x, y \in C$,

$$
H(T x, T y) \leq \alpha_{1}(d(x, T x)) d(x, T x)+\alpha_{2}(d(y, T y)) d(y, T y),
$$

where $a_{i}: \mathscr{B} \rightarrow[0,1)(i=1,2)$.

Then there exists a sequence $\left\{x_{n}\right\}$ in $C$ such that $T x_{n} \rightarrow D \in K(X)$ and each $y$ in $C \cap D$ satisfies $y \in T y$.

PROOF. As in the proof of Theorem 2.1, we obtain an asymptotically $T$-regular sequence $\left\{x_{n}\right\}$ in $C$. By hypothesis in (2), we obtain

$$
H\left(T x_{n}, T x_{m}\right) \leq \alpha_{1}\left(d\left(x_{n}, T x_{n}\right)\right) d\left(x_{n}, T x_{n}\right)+\alpha_{2}\left(d\left(x_{m}, T x_{m}\right)\right) d\left(x_{m}, T x_{m}\right) .
$$

Therefore, $\left\{T x_{n}\right\}$ is a Cauchy sequence in $K(X)$. The space $(K(X), H)$ is complete, thus there exists a $D \in K(X)$ such that $\lim T x_{n}=D$. By assumption $C \cap D \neq \phi$. Let $y \in C \cap D$. Then

$$
\begin{aligned}
d(y, T y) & \leq H(D, T y)=\lim H\left(T x_{n}, T y\right) \\
& \leq \lim \left[\left(\alpha_{1}\left(d\left(x_{n}, T x_{n}\right) d\left(x_{n}, T x_{n}\right)+\alpha_{2}(d(y, T y)) d(y, T y)\right]\right.\right. \\
& \leq \alpha_{2}(d(y, T y)) d(y, T y) .
\end{aligned}
$$

It follows that $\left[1-\alpha_{2}(d(y, T y))\right] d(y, T y) \leq 0$. Thus $d(y, T y)=0$. It implies that $y \in T y$.

A general result for a class of noncontinuous multivalued operators is established below.

THEOREM 2.3. Let $C$ be a nonempty bounded closed subset of a Banach space $X$. Let $T: C \rightarrow K(X)$ be a weakly inward and ${ }^{*}$-nonexpansive map. If $C$ has a family $G$ satisfying condition (*), then there exists a sequence $\left\{x_{n}\right\} \in C$ such that $x_{n} \rightarrow y \in C$ and $y \in T y$ provided one of the following conditions holds: 
(i) Each asymptotically T-regular sequence is Cauchy.

(ii) $T$ is hemicompact.

(iii) The collection $\left\{F_{n}: F_{n}=\lambda_{n} P_{T}, \lambda_{n} \in(0,1)\right.$ with $\left.\lambda_{n} \rightarrow 1\right\}$ is collectively condensing.

PROof. For each $x \in C, T x$ is compact, the map $P_{T}: C \rightarrow 2^{x}$ is well defined and is nonexpansive by the *-nonexpansiveness of $T$ (see, for example, [27, Theorem 2]). As $T x$ is compact for each $x \in C, P_{T}(x)$ must be compact for any $x$. Clearly, $P_{T}(x) \subseteq T x \subseteq \operatorname{cl}\left(I_{C}(x)\right)$ for all $x \in C$. Hence $P_{T}: C \rightarrow 2^{X}$ is weakly inward. Consequently, there exists a sequence $\left\{x_{n}\right\}$ in $C$ such that $d\left(x_{n}, P_{T} x_{n}\right) \rightarrow 0$ as $n \rightarrow \infty$. (i) A sequence is asymptotically $T$-regular if and only if it is asymptotically $P_{T}$-regular follows from the following inequalities

$$
d\left(x, P_{T}(x)\right) \leq d\left(x, u_{x}\right)=d(x, T x) \leq d\left(x, P_{T}(x)\right) \text { for all } x \in C .
$$

By Theorem 2.1 (a), there is a sequence $\left\{x_{n}\right\}$ in $C$ converging to $y$ such that $y \in P_{T}(y)$ and hence $y \in T y$.

(ii) From (3), we obtain $d\left(x_{n}, T x_{n}\right)=d\left(x_{n}, P_{T} x_{n}\right)$ for any sequence $\left\{x_{n}\right\} \in C$. Thus $T$ is hemicompact if and only if $P_{T}$ is hemicompact. So $y \in T y$ holds by Theorem 2.1 (b). (iii) Define the map $F: C \rightarrow 2^{X}$ by $F x=\bigcup_{n} P_{T_{n}} x, x \in C$. As in Theorem 2.1 (c), we get a sequence $\left\{x_{n}\right\}$ in $C$ such that $x_{n} \rightarrow y \in C$ and $y \in T y$.

THEOREM 2.4. Let $C, X, G$ and $T$ be as in Theorem 2.3. Suppose inequality (2) of Theorem 2.2 holds. Then there exists a sequence $\left\{x_{n}\right\} \in C$ such that $T x_{n} \rightarrow D \in$ $K(X)$ and each $y$ in $C \cap D$ satisfies $y \in T y$.

PROOF. As in the proof of Theorem 2.3, there exists a sequence $\left\{x_{n}\right\}$ in $C$ such that $d\left(x_{n}, P_{T} x_{n}\right) \rightarrow 0$ as $n \rightarrow \infty$. By (3), we get that $d\left(x_{n}, T_{x_{n}}\right) \rightarrow 0$ as $n \rightarrow \infty$. The rest of the proof is same as that of Theorem 2.2.

\section{Random solution}

In this section $(\Omega, A, \mu)$ denotes a complete $\sigma$-finite measure space unless stated otherwise. The following result will be needed.

THEOREM B ([28, Lemma 3.1]). Let $C$ be a closed subset of a complete separable metric space $X$ and $T: \Omega \times C \rightarrow C(X)$ a $d$-continuous random mapping. Then $T$ has a random fixed point (a random solution to $y(\omega) \in T(\omega, y(\omega)$ ) exists) if and only if $T$ has a generalized fixed point (a generalized solution to $y(\omega) \in T(\omega, y(\omega)$ ) exists). 
THEOREM 3.1. Let $C$ be a nonempty closed bounded subset of a separable Banach space $X$ and $T: \Omega \times C \rightarrow K(X)$ a weakly inward nonexpansive random mapping. Suppose that $C$ has a family $G$ satisfying condition (*). Then there exists a sequence $\left\{\xi_{n}\right\}$ of measurable mappings such that $\left\{\xi_{n}(\omega)\right\}$ is asymptotically $T(\omega, \cdot)$-regular, $\xi_{n}(\omega) \rightarrow \xi(\omega)$ (say) and $\xi(\omega) \in T(\omega, \xi(\omega)$ ) for each $\omega \in \Omega$ provided one of the following conditions holds:

(i) Each asymptotically $T(\omega, \cdot)$-regular sequence in $C$ is Cauchy for each $\omega \in \Omega$.

(ii) For each $\omega \in \Omega, T(\omega, \cdot)$ is hemicompact.

(iii) The collection $\left\{F_{n}(\omega, \cdot): F_{n}(\omega, \cdot)=\lambda_{n} T(\omega, \cdot), \lambda_{n} \in(0,1), \lambda_{n} \rightarrow 1\right\}$ is collectively condensing (collectively $k$-contractive, $0 \leq k<1$ ) for each $\omega \in \Omega$.

PROOF. As in the proof of Theorem 2.1, define $T_{n}: \Omega \times C \rightarrow K(X)$ by $T_{n}(\omega, x)=$ $\lambda_{n} T(\omega, x)$. Clearly each $T_{n}$ is a weakly inward contraction random operator. So each random operator inclusion $y_{n}(\omega) \in T_{n}\left(\omega, y_{n}(\omega)\right)$ has a random solution $\xi_{n}(\omega)$ by Theorem B.

By definition, $\xi_{n}(\omega)=\lambda_{n} \eta_{n}(\omega)$ where $\eta_{n}(\omega) \in T\left(w, \xi_{n}(\omega)\right.$ ) (see [3]). Moreover, for each $\omega \in \Omega,\left\{\eta_{n}(\omega)\right\}$ is bounded and so we obtain

$$
\left\|\xi_{n}(\omega)-\eta_{n}(\omega)\right\|=\left(1-\frac{1}{\lambda_{n}}\right)\left\|\eta_{n}(\omega)\right\| \rightarrow 0 \text { as } n \rightarrow \infty
$$

This implies that $\left\{\xi_{n}(\omega)\right\}$ is an asymptotically $T(\omega, \cdot)$-regular sequence for each $w \in \Omega$.

(i) By assumption $\left\{\xi_{n}(\omega)\right\}$ is a Cauchy sequence in $C$ for each $\omega \in \Omega$ and hence convergent. Thus $\xi(\omega)=\lim _{n} \xi_{n}(\omega)$, being the limit of measurable functions is measurable. The $T(\omega, \cdot)$-regularity of $\left\{\xi_{n}(\omega)\right\}$ and continuity of the function $x \rightarrow$ $d(x, T(\omega, x))$ imply that $\xi(\omega) \in T(\omega, \xi(\omega))$ for each $\omega \in \Omega$ as desired.

Similarly we can establish parts (ii) and (iii) by using corresponding deterministic parts of Theorem 2.1 and Theorem $B$.

COROLlaRY 3.2 ([4, Corollary 6]). Let $C$ be a compact convex subset of a separable Banach space $X$ and $T: \Omega \times C \rightarrow K(X)$ a weakly inward nonexpansive mapping. Then $y(\omega) \in T(\omega, y(\omega))$ has a random solution for each $\omega \in \Omega$.

Similar results have been obtained by Tan and Yuan [23, Theorem 2.6 and Theorem 2.7] where $A$ is a Suslin family and $X$ is a uniformly convex Banach space. Following is a random version of Theorem 2.3 (see also [11]).

THEOREM 3.3. Let $C$ be a nonempty closed bounded subset of a separable Banach space $X$. Let $T: \Omega \times C \rightarrow K(X)$ be a weakly inward d-continuous *-nonexpansive random operator. If $C$ has a family $G$ satisfying condition (*), then there is a 
sequence $\left\{\xi_{n}\right\}$ of measurable mappings such that $\left\{\xi_{n}(\omega)\right\}$ is asymptotically $T(\omega, \cdot)$ regular, $\xi_{n}(\omega) \rightarrow \xi(\omega)$ (say) and $\xi(\omega)$ is a random solution to $y(\omega) \in T(\omega, y(\omega)$ ) for each $\omega \in \Omega$ in the following cases:

(i) Each asymptotically $T(\omega, \cdot)$-regular sequence is Cauchy for each $w \in \Omega$.

(ii) $T(\omega, \cdot)$ is hemicompact for each $\omega \in \Omega$.

(iii) The collection $\left\{F_{n}(\omega, \cdot): F_{n}(\omega, \cdot)=\lambda_{n} P_{T}(\omega, \cdot), \lambda_{n} \in(0,1)\right.$ with $\left.\lambda_{n} \rightarrow 1\right\}$ is collectively condensing ( $k$-contractive, $0 \leq k<1$ ) for each $\omega \in \Omega$.

PROOF. Follows from Theorem 2.3 and Theorem B.

The following result can be easily verified.

THEOREM 3.4. Let $C, X, G$ and $T$ be as in Theorem 3.3. Suppose the following condition holds: $C \cap D \neq \phi$ for all $D \in K(X)$ with $D=\lim _{n} T\left(\omega, x_{n}\right)$ for some asymptotically $T(\omega, \cdot)$-regular sequence $\left\{x_{n}\right\} \in C$ and $T(\omega, \cdot)$ satisfies inequality (2) for all $x, y \in C$ and each $\omega \in \Omega$. Then there exists a measurable map $\xi: \Omega \rightarrow C$ such that $\xi(\omega) \in T(\omega, \xi(\omega))$ for each $\omega \in \Omega$.

Theorem 3.1 (ii) remains valid in the context of an arbitrary measure space $(\Omega, A, \mu)$; a reformulation of the same would be as follows:

THEOREM 3.5. Let $(\Omega, A, \mu)$ be an arbitrary measure space, $C$ a nonempty bounded and closed subset of a separable Banach space $X$ and $T: \Omega \times C \rightarrow K(X)$ a weakly inward hemicompact nonexpansive random mapping. Suppose that $C$ has a family $G$ satisfying condition (*). Then there is a sequence $\left\{\xi_{n}\right\}$ of measurable mappings such that $\left\{\xi_{n}(\omega)\right\}$ is asymptotically $T(\omega, \cdot)$-regular, $\xi_{n}(\omega) \rightarrow \xi(\omega)$ (say) and $\xi(\omega)$ is a random solution to $y(\omega) \in T(\omega, y(\omega))$ for each $\omega \in \Omega$.

PROOF. For each $\omega \in \Omega, y(\omega) \in T(\omega, y(\omega))$ has a generalized solution by Theorem 2.1 (b). The result now follows from [24, Theorem 2.3].

Every multivalued condensing map defined on a bounded closed subset of a complete metric space is hemicompact (see, for example, [24, Lemma 2.1]). Thus we have obtained in Theorem 3.5, multivalued analogues of Itoh [10, Theorem 2.1] and $\mathrm{Xu}[26$, Theorem 2 (i)].

COROLlaRY 3.6. Let $(\Omega, A, \mu)$ be any measure space, $C$ a nonempty bounded closed subset of a separable Banach space $X$ and $T: \Omega \times C \rightarrow K(X)$ a weakly inward condensing nonexpansive random mapping. Suppose that $C$ has a family $G$ satisfying condition (*). Then there is a sequence $\left\{\xi_{n}\right\}$ of measurable mappings such that $\left\{\xi_{n}(\omega)\right\}$ is asymptotically $T(\omega, \cdot)$-regular, $\xi_{n}(\omega) \rightarrow \xi(\omega)$ (say) and $\xi(\omega)$ is a random solution to $y(\omega) \in T(\omega, y(\omega))$ for each $\omega \in \Omega$. 
REMARKS 3.7. (i) Theorems 2.1-2.4, Theorem 3.1, Theorems 3.3-3.4 and Corollary 3.6 hold if we assume that $C$ is a starshaped subset of $X$.

(ii) Results similar to ours have been proved by $O^{\prime} \operatorname{Regan}[16]$ for a closed convex domain in the special Banach spaces $C([0, a], E)$ or $L^{p}([0, a], E), 1 \leq p<+\infty$, where $E$ is a given Banach space.

\section{Random fixed points on unbounded sets}

The interplay between fixed point and approximation results is interesting (see, for example, Lin [15]). In this section we prove a random fixed point theorem for *-nonexpansive operators defined on an unbounded set which in turn implies an approximation result for this class of operators. Further, we find random fixed points from the set of best approximations under a number of boundary conditions.

Following recent result due to Shahzad and Latif [22, Theorem 3.1] will be needed.

THEOREM C. Let $S$ be a nonempty separable closed subset of a complete metric space $X$ and $T: \Omega \times S \rightarrow X$ a continuous random operator for which condition (A) holds. If the set $G(\omega)=\{x \in S: x=T(\omega, x)\}$ is nonempty for each $\omega \in \Omega$, then $T$ has a random fixed point.

Using Theorem $\mathrm{C}$, we first establish an extension of [11, Theorem 2.1] to unbounded sets in a uniformly convex Banach space.

THEOREM 4.1. Let $C$ be a nonempty separable closed convex subset of a uniformly convex Banach space $X$ and $T: \Omega \times X \rightarrow 2^{X}$ a closed convex valued *-nonexpansive random operator for which condition (A) holds. Suppose $C$ is (KR)-bounded w.r.t. $T(\omega, \cdot)$ for each $\omega \in \Omega$ and $T$ either

(a) is weakly inward or

(b) satisfies Leray-Schauder condition.

If $P_{T}$ is a random operator, then $T$ has a random fixed point.

ProOF. A closed convex set in a uniformly convex Banach space is Chebyshev so each set $T(\omega, x)$ is Chebyshev. Thus for all $\omega \in \Omega, x \in C,\left\{u_{x}\right\}=P_{T}(\omega, x) \in$ $T(\omega, x)$. Thus

$$
d\left(P_{T}(\omega, x), P_{T}(\omega, y)\right)=d\left(u_{x}, u_{y}\right) \leq d(x, y) \quad \text { for all } x, y \in C \text { and } \omega \in \Omega .
$$

This implies that $P_{T}: \Omega \times C \rightarrow X$ is a nonexpansive random operator. As $P_{T}(\omega, x) \in$ $T(\omega, x) \subseteq \operatorname{cl}\left(I_{C}(x)\right)$ for all $x \in C$ and $\omega \in \Omega$, it follows that $P_{T}$ is weakly inward. Clearly $P_{T}$ satisfies the Leray-Schauder condition. Further (KR)-boundedness of $C$ 
with respect to $T(\omega, \cdot)$ implies that $C$ is (KR)-bounded with respect to $P_{T}(\omega, \cdot)$ for each $\omega \in \Omega$. Thus in both cases the set $G(\omega)=\left\{x \in C: x=P_{T}(\omega, x)\right\}$ is nonempty for each $\omega \in \Omega$ by [18, Theorem A]. By definition of $P_{T}$, we have for each $x \in C$ and for each $\omega \in \Omega$,

$$
d\left(x, P_{T}(w, x)\right)=d\left(x, u_{x}\right)=d(x, T(w, x)) \leq d\left(x, P_{T}(w, x)\right) .
$$

Therefore for any sequence $\left\{x_{n}\right\}$ in $C, D \in C(C)$ such that $d\left(x_{n}, D\right) \rightarrow 0$ and $d\left(x_{n}, P_{T}\left(w, x_{n}\right)\right) \rightarrow 0$, we obtain by (4) that $d\left(x_{n}, T\left(\omega, x_{n}\right)\right) \rightarrow 0$. As $T(\omega, \cdot)$ satisfies condition (A) so there exists $y \in D$ such that $y \in T(\omega, y)$. By (4), $d\left(y, P_{T}(\omega, y)\right)=d(y, T(\omega, y))=0$ and so $y=P_{T}(w y)$ for each $\omega \in \Omega$. Thus $P_{T}(\omega, \cdot)$ satisfies condition (A) for each $\omega \in \Omega$. By Theorem C, $P_{T}$ has a random fixed point. That is there exists a measurable map $\xi: \Omega \rightarrow C$ such that $\xi(\omega)=P_{T}(\omega, \xi(\omega))$ for each $\omega \in \Omega$. Since $P_{T}(\omega, \xi(\omega)) \in T(\omega, \xi(\omega))$ for each $\omega \in \Omega$, $\xi$ is the required random fixed point of $T$.

For single valued map the concepts of nonexpansive and *-nonexpansive coincide so the following corollary constitutes an extension of [10, Theorem 2.6], [14, Theorem 6 (ii)], [21, Corollary 3.4], [25, Corollary 3.5] and [26, Theorem 4] for unbounded sets.

COROLLARY 4.2. Let $C$ be a nonempty separable closed convex subset of a uniformly convex Banach space $X$ and $T: \Omega \times C \rightarrow X$ a nonexpansive random operator for which condition (A) holds. Suppose $C$ is (KR)-bounded w.r.t. $T(\omega, \cdot)$ for each $\omega \in \Omega$. If either $T:$ (a) is weakly inward or (b) satisfies the Leray-Schauder condition, then $T$ has a random fixed point.

As the condition (A) is always true for continuous condensing map $T: \Omega \times C \rightarrow X$ so if $T$ is a nonexpansive condensing random operator in Corollary 4.2 , then the conclusion of $[14$, Theorem 5 (ii)] is obtained with unbounded domain and range.

Let $C$ be a nonempty closed convex subset of a Hilbert space $X$. For every $T: C \rightarrow C K(X)$, we define $F: C \rightarrow C K(X)$ by $F x=\{y \in T x: d(y, C)=$ $d(T x, C)\}$. It is well known (see [20, page 535]) that $F x$ is nonempty as $T x$ is compact. As an application of Theorem 4.1, we establish a random approximation result for *-nonexpansive multivalued maps as follows.

THEOREM 4.3. Let $C$ be a nonempty separable closed convex subset of a Hilbert space $X$ and $T: \Omega \times C \rightarrow C K(X) a$ *nonexpansive random operator such that for each $\omega \in \Omega, F(\omega, \cdot)$ is *-nonexpansive and $\operatorname{PoF}(\omega, \cdot)$ satisfies condition (A), where $P$ stands for the proximity map on $C$. If there exists $x_{o} \in C$ such that for each $\omega \in \Omega, G\left(x_{o}, T\left(\omega, x_{o}\right) ; d\left(T\left(\omega, x_{o}\right), C\right) ; C\right)$ is bounded, then there exists a measurable 
map $\xi: \Omega \rightarrow C$ such that $d(\xi(\omega), T(\omega, \xi(\omega)))=d(T(\omega, \xi(\omega)), C)$ for each $\omega \in \Omega$ provided one of the following conditions holds:

(i) $P_{\mathrm{PoF}}$ is a random operator and for each $\omega \in \Omega, x \in C$ and $u_{x} \in F(\omega, x)$, with $d\left(x, P u_{x}\right)=d(x, \operatorname{PoF}(\omega, x))$, we have $d\left(x, u_{x}\right)=d(x, F(\omega, x))$.

(ii) $P_{F}$ is a random operator.

Proof. By definition,

$$
F(\omega, x)=\{v \in T(\omega, x):\|v-P v\|=d(T(\omega, x), C)\} .
$$

As $T$ is compact convex valued so $F$ and $\mathrm{PoF}$ are also compact convex valued multifunctions. We first show that $C$ is (KR)-bounded with respect to $\operatorname{PoF}(\omega, \cdot)$ for each $\omega \in \Omega$. Let $z \in G\left(x_{0}, \operatorname{PoF}\left(\omega, x_{0}\right) ; C\right)$. Then $z \in C$ and there exists $y \in \operatorname{PoF}\left(\omega, x_{0}\right)$ such that

$$
\|z-y\| \leq\left\|z-x_{0}\right\| .
$$

Since for any $\omega \in \Omega, y \in \operatorname{PoF}\left(\omega, x_{0}\right)$, there exists $u \in F\left(\omega, x_{0}\right) \subseteq T\left(\omega, x_{0}\right)$ such that $y=P u$. From (5) and (6) we obtain

$$
\|z-u\| \leq\|z-P u\|+\|P u-u\| \leq\left\|z-x_{0}\right\|+d\left(T\left(\omega, x_{0}\right), C\right) .
$$

It implies that $z \in G\left(x_{0}, T\left(\omega, x_{0}\right) ; d\left(T\left(\omega, x_{0}\right), C\right) ; C\right)$ where the last set is bounded for each $\omega \in \Omega$. Hence $C$ is (KR)-bounded w.r.t. $\operatorname{PoF}(\omega, \cdot)$ for each $\omega \in \Omega$.

(i) For any $\omega \in \Omega, x, y \in C$ and $u_{x} \in F x$ with $d\left(x, P u_{x}\right)=d(x, P o F(\omega, x))$, we have by the condition (i) that $d\left(x, u_{x}\right)=d(x, F(\omega, x))$. Since $F(\omega, \cdot)$ is *nonexpansive so for each $y \in C$, there is a unique $u_{y} \in F(\omega, y)$ with

$$
d\left(y, u_{y}\right)=d(y, F(\omega, y)) \text { and } d\left(u_{x}, u_{y}\right) \leq d(x, y) .
$$

As $\operatorname{PoF}(\omega, y)$ is closed and convex for each $\omega \in \Omega$ and $y \in C$, there exists a unique $P v_{y} \in \operatorname{PoF}(\omega, y)$ such that for each $\omega \in \Omega, y \in C, d\left(y, P v_{y}\right)=d(y, \operatorname{PoF}(\omega, y))$. Again by the condition (i), $d\left(y, v_{y}\right)=d(y, F(\omega, y))$. Uniqueness of $u_{y}$ in (7) implies that $v_{y}=u_{y}$ for each $y \in C$. Hence by nonexpansiveness of $P$, we obtain $d\left(P u_{x}, P u_{y}\right) \leq d\left(u_{x}, u_{y}\right) \leq d(x, y)$.

Thus PoF : $\Omega \times C \rightarrow C K(C)$ is a *-nonexpansive random operator. All the conditions of Theorem 4.1 are satisfied so PoF has a random fixed point. That is there exists a measurable map $\xi: \Omega \rightarrow C$ such that $\xi(\omega) \in \operatorname{PoF}(\omega, \xi(\omega))$ for $\omega \in \Omega$. Therefore, there is $v \in F(w, \xi(w)) \subseteq T(w, \xi(w))$ such that $\xi(w)=P v$. Hence for each $\omega \in \Omega$,

$$
\begin{aligned}
d(T(\omega, \xi(\omega)), C) & \leq d(\xi(\omega), T(\omega, \xi(\omega)) \leq\|\xi(\omega)-v\| \\
& =\|P v-v\|=d(T(\omega, \xi(\omega)), C)
\end{aligned}
$$


(ii) Since each set $F(\omega, x)$ is Chebyshev, so for all $\omega \in \Omega$ and $x \in C,\left\{u_{x}\right\}=$ $P_{F}(\omega, x) \in F(\omega, x)$. From the ${ }^{*}$-nonexpansiveness of $F$, we obtain

$$
d\left(P_{F}(\omega, x), P_{F}(\omega, y)\right)=d\left(u_{x}, u_{y}\right) \leq d(x, y)
$$

for each $x, y \in C$ and $\omega \in \Omega$. It implies that $P_{F}: \Omega \times C \rightarrow X$ is a nonexpansive random operator. Since $C$ is $(\mathrm{KR})$-bounded w.r.t. $\operatorname{PoF}(\omega, \cdot), C$ is (KR)-bounded w.r.t. $\operatorname{PoP}_{F}(\omega, \cdot)$ for each $\omega \in \Omega$. We have shown in the proof of Theorem 4.1 by using (4) that $T(\omega, \cdot)$ satisfies condition (A) if and only if $P_{T}(\omega, \cdot)$ satisfies condition (A). Thus $\operatorname{PoP}_{F}(\omega, \cdot)$ satisfies condition (A) because $\operatorname{PoF}(\omega, \cdot)$ satisfies the same. Notice that $\operatorname{PoP}_{F}: \Omega \times C \rightarrow C$ is a nonexpansive random operator. By Corollary 4.2, it follows that $\mathrm{PoP}_{F}$ has a random fixed point. That there is a measurable map $\xi: \Omega \rightarrow C$ such that $\xi(\omega)=\operatorname{PoP}_{F}(\omega, \xi(\omega))$ for each $\omega \in \Omega$ and hence $\xi(\omega)=\operatorname{PoP}_{F}(\omega, \xi(\omega)) \in \operatorname{PoF}(\omega, \xi(\omega))$ for each $\omega \in \Omega$. As in case (i), we have $d(\xi(\omega), T(\omega, \xi(\omega)))=d(T(\omega, \xi(\omega)), C)$ for each $\omega \in \Omega$.

THEOREM 4.4. Suppose the hypotheses of Theorem 4.3 hold. Suppose that in addition one of the following conditions is also satisfied:

(c) $T(\omega, \xi \omega)) \cap C \neq \phi$ for all $\omega \in \Omega$.

(d) $T(\omega, x) \cap C \neq \phi$ for each $\omega \in \Omega$ and $b x \in \partial C$.

(e) $T$ is an inward map; that is, $T(\omega, x) \subseteq I_{C}(x)$, for each $\omega \in \Omega$ and $x \in C$.

(f) $P_{T}$ is a random operator and for each $w \in \Omega, x \in C$, there exists a number $\lambda$ (real or complex, depending on whether the vector space $X$ is real or complex) such that $|\lambda|<1$ and $\lambda x+(1-\lambda) T(\omega, x) \subseteq C$.

(g) $P_{T}$ is a random operator and for each $\omega \in \Omega$ and for each $x \in C$ with $x \notin T(\omega, x)$, there exists $y$, depending on $\omega$ and $x$, in $I_{C}(x)$ such that

$$
\|y-z\| \leq\|x-z\| \text { for all } z \in T(\omega, x) \text {. }
$$

(h) $P_{T}$ is a random operator and $T$ is weakly inward.

(i) $P_{T}$ is a random operator and for each $\omega \in \Omega$ and $u \in \partial C$ with $u=\operatorname{PoP}_{T}(\omega, u)$, we have $u=T(\omega, u)$.

(j) $P_{T}$ is a random operator and for each $\omega \in \Omega$ and $x \in \partial C$, there exists $y \in C$, such that $\|z-y\| \leq\|x-y\|$ for all $z \in T(\omega, x)$.

Then $\xi$ of parts (i) and (ii) of Theorem 4.3 is a random fixed point of $T$.

PROOF. (c) In each of the cases (i) and (ii) of Theorem 4.3, there exists a measurable $\operatorname{map} \xi: \Omega \rightarrow C$ such that $\xi(\omega) \in \operatorname{PoF}(\omega, \xi(\omega))$ for each $\omega \in \Omega$.

As $T(\omega, \xi(\omega)) \cap C \neq \phi$ for each $\omega \in \Omega$, there is $y \in T(\omega, \xi(\omega)) \cap C$ and

$$
0=d(y, C)=d(T(w, \xi(w)), C) .
$$


It follows from (8) that $y \in F(\omega, \xi(\omega))$ for each $\omega \in \Omega$. Thus if $y_{0} \in F(\omega, \xi(w))$, then $y_{0} \in T(\omega, \xi(\omega))$. It further implies that $y_{0} \in T(\omega, \xi(\omega)) \cap C$ for each $\omega \in \Omega$. Therefore (8) holds for each $y_{0} \in F(\omega, \xi(\omega))$ and so $F(\omega, \xi(\omega)) \subseteq C$. It implies that $\operatorname{PoF}(\omega, \xi(\omega))=F(\omega, \xi(\omega))$ for each $\omega \in \Omega$. Thus $\xi(\omega) \in \operatorname{PoF}(\omega, \xi(\omega))=$ $F(\omega, \xi \omega)) \subseteq T(\omega, \xi(\omega))$ for each $\omega \in \Omega$.

(d) Since $\xi(\omega) \in \operatorname{PoF}(\omega, \xi(\omega))$ for each $\omega \in \Omega$, there exists $y \in F(\omega, \xi(\omega))$ such that $P y=\xi(\omega)$. If $\xi(\omega) \notin \partial C$ for some $\omega \in \Omega$, then $y \in C$, that is, $\xi(\omega)=y \in F(\omega, \xi(\omega)) \subseteq T(\omega, \xi(\omega))$; while if $\xi(\omega) \in \partial C$, we have by our assumption that $T(\omega, \xi(\omega)) \cap C \neq \phi$. Thus we have $T(\omega, \xi(\omega)) \cap C \neq \phi$ for each $w \in \Omega$. So (c) holds and we are done.

(e) Suppose for some $\omega \in \Omega, T(\omega, \xi(\omega)) \cap C=\phi$. Choose $y \in T(\omega, \xi(\omega))$ such that $\| y-\xi(\omega)) \|=d(T(\omega, \xi(\omega)), \xi(\omega))$.

Since $y \in I_{C}(\xi(\omega))$, there exist $z \in C$ and $\gamma>0$ such that $y=\xi(\omega)+\gamma(z-\xi(\omega))$. Now $y \notin C$, we can assume that $\gamma>1$. Then

$$
z=y / \gamma+(1-1 / r) \xi(w)=(1-\beta) y+\beta \xi(w),
$$

where $\beta=(1-1 / r), 0<\beta<1$. Thus

$$
\begin{aligned}
d(T(\omega, \xi(\omega)), C) & \leq \| y-((1-\beta) y+\beta \xi \omega)) \| \\
& =\beta \| y-\xi(\omega \|<d(T(\omega, \xi(\omega)), C),
\end{aligned}
$$

which is impossible. Thus, for each $\omega \in \Omega, T(\omega, \xi(\omega)) \cap C \neq \phi$. So (c) must hold. (f) By definition of $P_{T}$, we have $P_{T}(\omega, x)=\left\{u_{x}\right\} \in T(\omega, x)$ and

$$
d\left(x, P_{T}(\omega, x)\right)=d\left(x, u_{x}\right)=d(x, T(\omega, x)) \leq d\left(x, P_{T}(\omega, x)\right)
$$

for each $\omega \in \Omega, x \in C$. Thus it follows from $d(\xi(\omega), T(\omega), \xi(\omega))=d(T(\omega, \xi(\omega)), C)$ that $d\left(\xi(\omega), P_{T}(\omega, \xi(\omega))\right)=d\left(P_{T}(\omega, \xi(\omega)), C\right)$ for each $\omega \in \Omega$. As $P_{T}(\omega, x) \in$ $T(\omega, x), \lambda x+(1-\lambda) P_{T}(\omega, x) \in C$ for each $\omega \in \Omega, x \in C$ and given $\lambda$ in (f). Hence $P_{T}$ and so $T$ has a random fixed point by [25, Theorem 4.9 (i)].

Similarly the parts $(\mathrm{g})-(\mathrm{j})$ follow from the corresponding parts of $[25$, Theorem 4.9 (ii)-(v)] respectively (see the proof of [5, Theorem 4.1]).

For single valued map the concepts of *-nonexpansive and nonexpansive coincide and the conditions (i) and (ii) in Theorem 4.3 are redundant. The continuous hemicompact maps satisfy condition (A). Thus the conclusion of [25, Theorem 4.3] for nonexpansive random operator $T$ without the assumptions: for each $\omega \in \Omega, T(\omega, C)$ is bounded and I-PoT $(\omega, \cdot)(C)$ is a closed subset of $X$ follows from the following:

COROLLARY 4.5. Let $C$ be a nonempty separable closed convex subset of a Hilbert space $X$ and $T: \Omega \times C \rightarrow X$ a nonexpansive random operator such that $\operatorname{PoT}(\omega, \cdot)$ is 
hemicompact for each $\omega \in \Omega$. Suppose there exists $x_{0} \in C$ such that for each $\omega \in \Omega$, $G\left(x_{0}, T\left(\omega, x_{0}\right), d\left(T\left(\omega, x_{0}\right), C\right) ; C\right)$ is bounded. Then there exists a measurable map $\xi: \Omega \rightarrow C$ such that for each $\omega \in \Omega,\|\xi(\omega)-T(\omega, \xi(\omega))\|=d(T(\omega, \xi(\omega)), C)$.

For single valued map $T$, we have $P_{T}=T$. Thus Theorem 4.4 reduces to the following result which includes [25, Theorem 4.9] for nonexpansive random operators.

COROLlARY 4.6. Let $C$ be a nonempty separable closed convex subset of a Hilbert space $X$ and $T: \Omega \times C \rightarrow X$ a nonexpansive random operator such that $\operatorname{PoT}(\omega, \cdot)$ is hemicompact for each $\omega \in \Omega$. Suppose there is $x_{0} \in C$ such that for each $\omega \in \Omega, G\left(x_{0} ; T\left(w, x_{0}\right) ; d\left(T\left(w, x_{0}\right), C\right) ; C\right)$ is bounded and $T$ satisfies any one of the conditions $(\mathrm{f})-(\mathrm{j})$ in Theorem 4.4. Then $T$ has a random fixed point.

\section{Acknowledgements}

The first author work is supported by Kuwait University Research Grant SM 03/00. The second author acknowledges gratefully the support provided by King Fahd University of Petroleum and Minerals during this research.

\section{References}

[1] D. E. Anderson, J. L. Nelson and K. L. Singh, 'Fixed points for single and multivalued mappings in locally convex spaces', Math. Japon. 31 (1986), 665-672.

[2] I. Beg, 'Random fixed points of non-self maps and random approximations', J. Appl. Math. Stochastic Anal. 102 (1997), 127-130.

[3] I. Beg and N. Shahzad, 'Random fixed point theorems for nonexpansive and contractive-type random operators on Banach spaces', J. Appl. Math. Stochastic Anal. 7 (1994), 569-580.

[4] — , 'Random fixed point theorems for multivalued inward random operators on Banach spaces', Adv. Math. Sci. Appl. 5 (1995), 31-37.

[5] - 'Applications of the proximity map to random fixed point theorems in Hilbert spaces', $J$. Math. Anal. Appl. 196 (1995), 606-613.

[6] - 'On random approximations and a random fixed point theorem for multivalued mappings defined on unbounded sets in Hilbert spaces', Stochastic Anal. Appl. 14 (1996), 507-511.

[7] A. Canetti, G. Marino and P. Pietramala, 'Fixed point theorems for multivalued mappings in Banach spaces', Nonlinear Anal. 17 (1991), 11-20.

[8] C. J. Himmelberg, 'Measurable relations', Fund. Math. 87 (1975), 53-72.

[9] T. Husain and A. Latif, 'Fixed points of multivalued nonexpansive maps', Math. Japon. 33 (1988), 385-391.

[10] S. Itoh, 'Random fixed point theorems with applications to random differential equations in Banach spaces', J. Math. Anal. Appl. 67 (1979), 261-273.

[11] A. R. Khan and N. Hussain, 'Random fixed points for *-nonexpansive random operators', J. Appl. Math. Stochastic Anal. 14 (2002), 341-349. 
[12] A. R. Khan, N. Hussain and A. B. Thaheem, 'Applications of fixed theorems to invariant approximation', Approx. Theory and its Appl. 16 (2000), 48-55.

[13] W. A. Kirk and W. O. Ray, 'Fixed point theorems for mappings defined on unbounded sets in Banach spaces', Studia Math. 64 (1979), 127-138.

[14] T. C. Lin, 'Random approximations and random fixed point theorems for non-self maps', Proc. Amer. Math. Soc. 103 (1988), 1129-1135.

[15] — 'Random approximations and random fixed point theorems for continuous 1-set constructive random maps', Proc. Amer. Math. Soc. 123 (1995), 1167-1176.

[16] D. O'Regan, 'Nonlinear operator approximation theory', Numer. Funct. Anal. Optimiz. 19 (1998), 587-592.

[17] N. S. Papageorgiou, 'Random fixed point theorems for measurable multifunctions in Banach spaces', Proc. Amer. Math. Soc. 97 (1986), 507-514.

[18] S. Park, 'Best approximations and fixed points of nonexpansive maps in Hilbert spaces', Numer. Funct. Anal. Optimiz. 18 (1997), 649-657.

[19] V. M. Sehgal and S. P. Singh, 'On random approximation and a random fixed point theorem for set-valued mappings', Proc. Amer. Math. Soc. 95 (1985), 91-94.

[20] —_, 'A generalization to multifunctions of Fan'best approximation theorem', Proc. Amer. Math. Soc. 102 (1988), 534-537.

[21] N. Shahzad, 'Random fixed point theorems for various classes of 1-set-contractive maps in Banach spaces', J. Math. Anal. Appl. 203 (1996), 712-718.

[22] N. Shahzad and S. Latif, 'Random fixed points for several classes of 1-ball-contractive and 1-setcontractive random maps', J. Math. Anal. Appl. 237 (1999), 83-92.

[23] K. K. Tan and X. Z. Yuan, 'On deterministic and random fixed points', Proc. Amer. Math. Soc. 119 (1993), 849-856.

[24] _ - 'Random fixed point theorems and approximation in Cones', J. Math. Anal. Appl. 185 (1994), 378-390.

[25] - 'Random fixed point theorems and approximation', Stochastic Anal. Appl. 15 (1997), 103-123.

[26] H. K. Xu, 'Some random fixed point theorems for condensing and nonexpansive operators', Proc. Amer. Math. Soc. 110 (1990), 395-400.

[27] —, 'On weakly nonexpansive and *-nonexpansive multivalued mappings', Math. Japon. 36 (1991), 441-445.

[28] H. W. Yi and Y. C. Zhao, 'Fixed point theorems for weakly inward multivalued mappings and their randomizations', J. Math. Anal. Appl. 183 (1994), 613-619.

Lahore University of Management Sciences

KFUPM

Lahore

Dhahran

Pakistan

Saudi Arabia

e-mail: ibeg@lums.edu.pk

\author{
Bahauddin Zakariya University \\ Multan \\ Pakistan
}

